\title{
Clinical Case Reports as a Tool for Medical Student Education
}

\author{
Adeel Ashraf ${ }^{1}$, Nabeel Ashraf ${ }^{1}$, Taeok Bae ${ }^{2}$ \\ ${ }^{1}$ Indiana University School of Medicine; ${ }^{2}$ Department of Microbiology and Immunology \\ Indiana University School of Medicine
}

Background: The first two years of medical student education relies on textbooks and lectures for a foundation of knowledge that must be expounded upon in the latter two years. However, this doesn't mean that students should be constrained to a narrow usage of their intelligence for exam questions, rather they can begin to think about using their knowledge in a clinical setting from the beginning. We researched eight prevalent pathogens that have a higher probability of being encountered by a physician by using real life examples from case reports from around the world. We condensed the materials into a team-based learning (TBL) activity with thought provoking questions related to the case in particular and to fundamental principles of microbiology and immunology as a whole.

Approach: We searched extensively through online databases to locate clinical case reports that weren't too uncommon or too simple. Next, we built a TBL activity using the case report as the framework with clinical presentation, lab values, and images in a certain order that would resemble the way that the physician encountered it. A mixture of questions from simple identification to complex mechanisms were asked along with review of other similar pathogens and relevant pharmacology. Along with every activity, a small abridged review of the pathogen was written after researching and consulting appropriate literature. The review included the most clinically significant information to supplement the TBL activity such as history, epidemiology, transmission, disease, diagnosis, and treatment of the pathogen.

Future implications: These TBL activities can be utilized in future host defense course as a way for students to test their understanding while exploring a more clinically relevant scenario. Hopefully, students will appreciate the real world application of their expansive knowledge, and apply their newly developed understanding in rotations, residency, and even their medical practice. 